\title{
Limited Invasive Techniques in Management of Sander's Type II, III Calcaneal Fractures
}

\author{
Ahmad Atef Abou Elsoud*, Adel Mohammad Salama, Amr Mohammad Al Adawy \\ Orthopedic Surgery Department, Faculty of Medicine, Zagazig University, Egypt \\ *Corresponding author: Ahmad A. Abou Elsoud, Mobile: (+2)0111631931, Email: ahmedatefaboelsoud@gmail.com
}

\begin{abstract}
Background: A thorough understanding of the clinical and radiographic anatomy of the calcaneus and its articulations is crucial when attempting less invasive procedures for intraarticular calcaneus fractures.

Objective: The aim of this work is to evaluate the functional outcome and to assess the anatomical restoration of the calcaneus with radiographic measurements after percutaneous fixation of Sanders type II and type III calcaneal fractures by K-wires and cannulated screws.

Patients and methods: This was a prospective study that is conducted on 23 patients whom were classified as Sanders type II, III Calcaneal fractures. This study was done at Alexandria Police Hospital and Zagazig University Hospital. These patients were followed up for at least 6 months postoperatively. All patients in the study were evaluated including history taking and clinical examination. All patients had preoperative lab investigations and were evaluated radiologically with X-ray calcaneus and $\mathrm{CT}$ scan, and were followed up at outpatient clinic twice in the first week then once a week for the next two months.

Results: The final results at the end of this study were satisfactory in $91.3 \%$ of patients and unsatisfactory in $8.7 \%$ of patients. About (81.3\%) of the excellent results were obtained in Sanders type II fractures, while $18.7 \%$ of the excellent results were obtained in Sanders type III fractures.

Conclusion: Less invasive surgical techniques for treating displaced intraarticular calcaneus fractures have been undertaken in an attempt to reduce complications and improve recovery when surgery is indicated. These emerging techniques may be beneficial in patients with soft-tissue compromise, multiple comorbidities, and displaced intraarticular fractures with minimal comminution.
\end{abstract}

Keywords: Calcaneal fractures, Cannulated screws, K- wires, Percutaneous fixation.

\section{INTRODUCTION}

Fractures of the calcaneus account for approximately $60 \%$ of tarsal injuries and usually are the result of a fall from a height ${ }^{(\mathbf{1})}$. More than $70 \%$ of calcaneal fractures are intraarticular involving the subtalar joint, mostly caused by a fall from a height with the heel directly hitting the ground ${ }^{(2)}$.

Over $80 \%$ of calcaneal fractures involve the posterior articular surface (PAS) of the bone resulting in incongruence of the subtalar joint. This leads to arthritis of the joint with painful limitation of motion and intolerance of uneven surfaces. Increasing difficulty in dorsiflexion of the foot impedes gait leading to a limp. Reduction of the height of the calcaneal tuberosity leads to impingement of malleoli on the shoe sole and disorders of Achilles tendon mechanics, while compression of the peroneal tendons causes tendinopathy. Widening of the flattened heel increases compression and makes fitting standard shoes even more difficult ${ }^{(3,4)}$.

Infection rates after using the extended lateral approach vary between $1.3-7 \%$. Several smaller direct approaches to the lateral calcaneal wall, resembling the classical palmar approach, have been carried out without significantly reducing the soft tissue problems as they cut throw the lateral calcaneal artery ${ }^{(5)}$. A direct approach over the sinus tarsi requiring retraction of the peroneal tendons and sural nerve with minimal fixation resulted in an infection rate of $8.5 \%$ and further $9.2 \%$ soft tissue problem like peroneal tendinitis, sinus tarsi syndrome, and even compartment syndrome ${ }^{(\boldsymbol{6})}$.

To avoid the feared soft tissue complications, several minimally invasive and percutaneous approaches have been proposed throughout the history of calcaneal fracture treatment and recently gained popularity for selected injury patterns ${ }^{(7)}$. After the first percutaneous operation by the German surgeon Westhues, a considerable number of percutaneous and minimally invasive open techniques have been used to treat calcaneal fractures ${ }^{(\mathbf{8})}$.

Therefore, this study aimed to evaluate the functional outcome and to assess the anatomical restoration of the calcaneus with radiographic measurements after percutaneous fixation of Sanders type II and type III calcaneal fractures by K- wires and cannulated screws.

\section{PATIENTS AND METHODS}

This study was done at Alexandria Police Hospital and Zagazig University Hospital. These patients were followed up for at least 6 months postoperatively. Open, extra-articular of Sander's, type I and type IV fractures and patients with ipsilateral vascular injury or peripheral neuropathy were excluded from this study.

All patients in the study were evaluated including history taking and clinical examination. All 
patients has preoperative lab investigations and evaluated radiologically with X-ray calcaneus and CT scan.

\section{Surgical techniques:}

All patients were placed in lateral decubitus position with affected limb placed as uppermost limb on a radiolucent operating table, with a firm bump underneath the injured foot, which protruded outside the table, to support a perfect lateral position. All patients were placed in lateral position with affected limb placed as uppermost limb, lateral minimal invasive incision was done for elevation of the depressed segment, then fixation was done by K-wires or cannulated screws and all patients were put in below knee cast and followed up twice per week for the first week.

Then once a week for the next two months. Elevation of depressed articular surface was done using periosteum elevator inserted percutaneously by lateral incision under image under the depressed part from lateral to medial part. Under C-arm guidance, a Steinmann pin or Schanz was inserted through the calcaneal tuberosity directed from lateral to medial. Temporary fixation of elevated articular surface was done using percutaneous K-wire. Then permanent fixation of the depressed articular surface was done by $4 \mathrm{~mm}$ cannulated screw directed towards the sustentaculum tali. Then, drilling over K-wires was done by cannulated drill bit. The stab wounds were sutured with absorbable sutures and padded bandage applied.

\section{Postoperative Follow up:}

Postoperatively patients received intravenous injection of ceftriaxone $1 \mathrm{~g}$ twice per day for three days. Oral antibiotics were continued for three more days and then discontinued. The postoperative protocol employed an immediate postoperative radiograph (lateral and axial views) and measurement of the three angles: Gissane, Bohler's angle and posterior facet inclination angles. The patients started postoperative ankle mobilization with non-weight bearing axillary crutch mobilization. They were followed up at outpatient clinic twice in the first week then once a week for the next two months.

\section{Ethical approval:}

The study was approved by the Ethical Committee of Zagazig Faculty of Medicine. An informed consent was obtained from all patients in this research. Every patient received an explanation for the purpose of the study. All given data were used for the current medical research only. This work has been carried out in accordance with The Code of Ethics of the World Medical Association (Declaration of Helsinki) for studies involving humans.

\section{Statistical analysis}

Data were collected, tabulated and statistically analyzed by an IBM compatible personal computer with SPSS statistical package version 20. Qualitative data were presented as number (N) and percent (\%) and were compared by Chi square test or Fisher's exact test. Quantitative data were presented as mean \pm standard deviation (SD). Independent and paired Student t-test were used for comparison between two independent or paired groups respectively. $\mathrm{P}$ value < 0.05 was considered significant.

\section{RESULTS}

In this study, the mean age was 34.83 (range, 22-46) years old. There was male predominance as male:female ratio was 19:4. Majority of patients (65\%) had fracture calcaneus on the right side (Table 1).

Table (1): Demographic data among the studied patients

\begin{tabular}{|c|c|c|}
\hline Age group & Frequency & Percentage \\
\hline $21->30$ & 12 & $52 \%$ \\
\hline $30->40$ & 5 & $21 \%$ \\
\hline $40-50$ & 6 & $28 \%$ \\
\hline Total & 23 & $100 \%$ \\
\hline Sex group & No. of patients & Percentage \\
\hline Male & 19 & $83 \%$ \\
\hline Female & 4 & $17 \%$ \\
\hline Total & 23 & $100 \%$ \\
\hline Side & No. of patients & Percentage \\
\hline Right only & 15 & $65 \%$ \\
\hline Left only & 5 & $22 \%$ \\
\hline Bilateral & 3 & $13 \%$ \\
\hline Total & 23 & $100 \%$ \\
\hline
\end{tabular}

The final results at the end of this study were satisfactory in $91.3 \%$ of patients and unsatisfactory in $8.7 \%$ of patients (Table 2 ).

Table (2): The clinical results among the studied patients

\begin{tabular}{|l|c|c|}
\hline \multicolumn{1}{|c|}{ Results } & $\begin{array}{c}\text { Number of } \\
\text { cases }\end{array}$ & Percentage \\
\hline Satisfactory: & 16 & $69.6 \%$ \\
$\begin{array}{l}\text { Excellent } \\
\text { Good }\end{array}$ & 5 & $21.7 \%$ \\
\cline { 2 - 3 } $\begin{array}{l}\text { Unsatisfactory: } \\
\text { Fair } \\
\text { Poor }\end{array}$ & 2 & $91.3 \%$ \\
\hline \multicolumn{1}{|c|}{ Total } & 0 & $0.7 \%$ \\
\cline { 2 - 3 } & & $8.7 \%$ \\
\hline \multicolumn{2}{|c|}{ There was a statistically significant } \\
\hline
\end{tabular}

improvement of Bohler's angle after fixation (Table 3). 
Table (3): Bohler's angle before and after fixation ( 23 cases)

\begin{tabular}{|c|c|c|c|}
\hline $\begin{array}{c}\text { Bohler } \\
\text { angle }\end{array}$ & Mean & Range & P-value \\
\hline $\begin{array}{c}\text { Before } \\
\text { fixation }\end{array}$ & $\begin{array}{c}15.7 \\
\pm 3.5^{\circ}\end{array}$ & $8-22^{\circ}$ & \multirow{2}{*}{$\mathbf{0 . 0 0 0 3}$} \\
\cline { 1 - 3 } After & 26.5 & $20-$ & \\
fixation & $\pm 3.9^{\circ}$ & $35^{\circ}$ & \\
\hline
\end{tabular}

Restoration of normal range Bohler angle (25$40)^{\circ}$ was achieved in $65.2 \%$ of the patients (Table 4).

Table (4): Showing achievement of Bohler's angle after fixation

\begin{tabular}{|c|c|c|}
\hline Bohler angle & $\begin{array}{c}\text { Number of } \\
\text { patients }\end{array}$ & Percentage \\
\hline Achieved & 15 & $\mathbf{6 5 . 2 \%}$ \\
\hline Not achieved & $\mathbf{8}$ & $\mathbf{3 4 . 8 \%}$ \\
\hline Total & $\mathbf{2 3}$ & $\mathbf{1 0 0 \%}$ \\
\hline
\end{tabular}

Restoration of normal angles postoperatively was achieved in 13 patients (Table 5).

Table (5): Number of target angles achieved in relation to mechanism of injury

\begin{tabular}{|c|c|c|c|}
\hline $\begin{array}{l}\text { Number of target } \\
\text { angles achieved }\end{array}$ & FFH & RTA & P-value \\
\hline One angle & $11(84.6 \%)$ & $2(15.4 \%)$ & \multirow{3}{*}{0.599} \\
\hline Two angles & $5(100 \%)$ & O (0\%) & \\
\hline All 3 angles & $4(80.0 \%)$ & $1(20.0 \%)$ & \\
\hline
\end{tabular}

There was insignificant difference between satisfactory and unsatisfactory of end results as regard age, gender, mechanism of injury, and severity of fracture (Table 6).

Table (6): Relationship between demographic data and the end results ( 23 cases)

\begin{tabular}{|c|c|c|c|}
\hline & \multicolumn{3}{|c|}{ MFS 6 months after fixation } \\
\cline { 2 - 4 } & $\begin{array}{c}\text { Unsatisfactory } \\
(\mathbf{N = 2})\end{array}$ & $\begin{array}{c}\text { Satisfactory } \\
(\mathbf{N = 2 1})\end{array}$ & P-value \\
\hline Age & $24 \pm 1.0$ & $32.2 \pm 7.3$ & 0.135 \\
\hline Gender & $2(28.6 \%)$ & $21(100.0 \%)$ & 0.083 \\
\hline $\begin{array}{c}\text { Mechanism } \\
\text { of Injury }\end{array}$ & FFH & $2(100.0 \%)$ & 0.125 \\
\hline $\begin{array}{c}\text { Sander's } \\
\text { classification } \\
\text { Type II } \\
\text { Type III }\end{array}$ & $1(50 \%)$ & $16(71.4 \%)$ & 0.420 \\
\hline
\end{tabular}

\section{DISCUSSION}

The percutaneous and minimally invasive open techniques can minimize the incidence of soft tissue complications, one disadvantage might be the risk of incomplete reduction, especially in difficult intraarticular calcaneal fracture ${ }^{(8)}$. An incongruency in the posterior facet of the subtalar joint and failure to restore the angle of Bohler have been frequently mentioned as important predictors of outcome after operative treatment of calcaneal fractures ${ }^{(9)}$.

The present study included 23 patients with
Sanders type II, III calcaneal fractures for evaluation of the functional outcome and to assess the anatomical restoration of the calcaneus with radiographic measurements after percutaneous fixation of Sanders type II and type III calcaneal fractures by K- wires and cannulated screws.

Based on several studies with percutaneous techniques, Rammelt $\boldsymbol{e t} \boldsymbol{a l} .{ }^{(5)}$ concluded that the percutaneous fixation of displaced calcaneusfractures produces good to excellent results in properly selected patients with less severe fractures patterns and that the quality of joint reduction should be directly visualized to avoid problems in subtalar joint motion.

The current outcomes of our study is concur with Cao et al. (10) who reviewed 33 feet in 33 consecutive patients with Sanders type II and III calcaneal fractures who had undergone a minimally invasive technique using percutaneous reduction and locking plates. The median functional score of the patients reached 82 at the last follow-up evaluation according to the American Orthopedic Foot and Ankle Society ankle-hind foot scale. All cases achieved restoration of a normal Böhler's angle. This treatment seems to be more suitable for cases with moderately displaced fracture or non-comminuted calcaneus fracture because of the limited fixation strength ${ }^{(11)}$. For instance, Stulik et al. ${ }^{\left({ }^{12}\right)}$ reported 13 cases (4.5\%) of loss of reduction in patients treated with closed reduction and percutaneous fixation using $\mathrm{K}$-wires.

The development of major wound complications is a serious concern in treating calcaneal fractures as the soft tissue envelope around the calcaneus is particularly thin and vulnerable over the lateral wall, which is exposed for surgery in most cases. The hazard with the medial approaches lies in the nearby neurovascular bundle ${ }^{(13)}$. With combined medial and lateral approaches, the rate of wound edge necrosis reaches $27 \%{ }^{(14)}$. Minimally invasive sinus tarsi approach was also recommended by some authors due to fewer wound complications and easy subtalar articular reduction ${ }^{(15)}$.

Our results agree with Jin et al. (11) who concluded that minimally invasive percutaneous offers critical advantages, including a considerably reduced risk of wound healing complications, shortened operating time and length of hospital stay, as well as superior postoperative rehabilitation.

We achieved satisfactory anatomical reduction of the calcaneus in the studied patients. However, longer term studies with a larger sample size and more randomized controlled trials are required to define the superiority of our minimally invasive technique compared with conventional surgical treatment of calcaneal fractures.

\section{CONCLUSION}

Less invasive surgical techniques for treating displaced intraarticular calcaneus fractures have been undertaken in an attempt to reduce complications and 
improve recovery when surgery is indicated. These emerging techniques may be beneficial in patients with soft-tissue compromise, multiple comorbidities, and displaced intraarticular fractures with minimal comminution.

\section{Financial support and sponsorship: Nil. Conflict of interest: Nil.}

\section{REFERENCES}

1. Barei D, Bellabarba C, Sangeorzan B et al. (2002): Fractures of the calcaneus. Orthopedic Clinics, 33(1): 263-285.

2. Biz C, Barison E, Ruggieri $P$ et al. (2016): Radiographic and functional outcomes after displaced intra-articular calcaneal fractures: a comparative cohort study among the traditional open technique (ORIF) and percutaneous surgical procedures (PS). Journal of Orthopaedic Surgery and Research, 11(1): 1-11.

3. Zwipp H, Rammelt S, Grass R (2002): Ligamentous injuries about the ankle and subtalar joints. Clinics in Podiatric Medicine and Surgery, 19(2): 195-229.

4. Jomha N, Scharfenberger A, Goplen G et al. (2013): Ankle osteoarthritis and arthroplasty. https://www.intechopen.com/chapters/428 31

5. Rammelt S, Zwipp H (2004): Calcaneus fractures: facts, controversies and recent developments. Injury, 35(5): 443-461.

6. Rammelt $\mathbf{S}$, Hans $\mathbf{Z}$ (2006): Calcaneus fractures: facts, controversies and recent developments. Trauma, 8: 197212.

7. Khurana A, Dhillon M, Prabhakar S et al. (2017): Outcome evaluation of minimally invasive surgery versus extensile lateral approach in management of displaced intra-articular calcaneal fractures: a randomised control trial. The Foot, 31: 23-30.

8. van Hoeve S, Poeze M (2016): Outcome of minimally invasive open and percutaneous techniques for repair of calcaneal fractures: a systematic review. The Journal of Foot and Ankle Surgery, 55(6): 1256-1263.

9. Roukis T (2019): Closed manipulation, intraosseous reduction, and rigid internal fixation for displaced intraarticular calcaneal fractures. Clin Podiatry Med Surg., 36: 197-210.

10. Cao L, Weng W, Song $S$ et al. (2015): Surgical treatment of calcaneal fractures of Sanders type II and III by a minimally invasive technique using a locking plate. The Journal of Foot and Ankle Surgery, 54(1): 7681.

11. Jin C, Weng D, Yang W et al. (2017): Minimally invasive percutaneous osteosynthesis versus ORIF for Sanders type II and III calcaneal fractures: a prospective, randomized intervention trial. Journal of Orthopaedic Surgery and Research, 12(1): 1-9.

12. Stulik J, Stehlik J, Rysavy M et al. (2006): Minimallyinvasive treatment of intra-articular fractures of the calcaneum. J Bone Joint Surg., 88: 1634-41.

13. Lowery R, Calhoun J (1996). Fractures of the calcaneus part II: treatment. Foot \& Ankle International, 17(6): 360-366.

14. Wang $Q$, Chen W, Su Y et al. (2010): Minimally invasive treatment of calcaneal fracture by percutaneous leverage, anatomical plate, and compression bolts the clinical evaluation of cohort of 156 patients. Journal of Trauma and Acute Care Surgery, 69(6): 1515-1522.

15. Xia S, Wang X, Lu Y et al. (2013): A minimally invasive sinus tarsi approach with percutaneous plate and screw fixation for intra-articular calcaneal fractures. International Journal of Surgery, 11(10): $1087-$ 1091. 